\title{
SYMOBL-WISE INTERLEAVING FOR SERIALLY CONCATENATED CONTINUOUS PHASE MODULATION
}

\author{
Jun Ning and Minyue Fu \\ School of EECS, University of Newcastle, NSW 2308 Australia \\ Email: minyue.fu@newcastle.edu.au
}

\begin{abstract}
Serially concatenated continuous phase modulation (SCCPM) is known to be a preferred choice of modulation technique for achieving high efficiencies in bandwidth and power. Commonly used SCCPM schemes use bitwise interleaving for concatenations. In this paper, we consider symbolwise interleaving based on a decoding error analysis. We show that, by using an appropriate decoding algorithm, this symbol-wise scheme of SCCPM leads to considerable improvements in bit error rate (BER) performance over the scheme using bitwise interleaving.
\end{abstract}

Index Terms - Continuous phase modulation, serially concatenated CPM, APP decoding, symbol-wise interleaving.

\section{INTRODUCTION}

Continuous phase modulation (CPM) plays an important role in radio and satellite communications due to two crucial properties, namely, constant envelope and phase continuity $[1,2]$. The first property makes CPM a preferred choice where a nonlinear amplifier is employed. The second property, if utilized properly, makes CPM an efficient modulation scheme in both bandwidth and power.

The spectral efficiency of a CPM depends on a number of parameters. These include the shape function $g(t)$ (rectangular, raised cosine or Gaussian), the duration $L$ of the shape function, the symbol period $T$, the cardinality of the CPM symbols $M$, and the modulation index $h$. In this paper, the modulation index is restricted to the form of $h=1 / p$, where $p$ is the number of phase increments. A high spectral efficiency can be achieved by increasing the values of $L, M$ and $p$. This comes with the tradeoff of higher computational complexity, especially for decoding. To improve the power efficiency, a CPM system is typically cascaded with an outer code.

There are two common schemes for cascaded CPM. The first scheme uses the fact that a CPM can be decomposed into a continuous phase encoder (CPE) and memoryless modulator (MM) [5]. The cascading is done by directly combining the CPE with an outer code to form a single convolutional code (typically non-binary); see [3, 4, 6]. The advantage of this scheme is that a single trellis is needed for maximum likelihood decoding. The disadvantages, however, are that the performance improvements from cascading are limited and that the outer code needs to have the same algebraic structure as the inner code (CPM). The second scheme concatenates an outer code with the CPM through an interleaver. This type of cascaded CPM is often referred to as serially concatenated CPM (SCCPM); see [7]-[12]. We only study the SCCPM scheme in this paper because this scheme is known to give better performances.

A typical SCCPM consists of an binary outer code, an bitwise interleaver and an inner code (CPM) which is connected to the channel directly. When $M>2$, a mapper is also needed to bridge between the bitwise interleaver and the (non-binary) CPM. Iterative methods are typically used for decoding. A detailed a posterior probability (APP) decoding procedure is given in [17, 18]. It is inferred in [18] that the probability distribution of a CPM symbol can be represented as a product of the marginal distributions of the $\log _{2} M$ bits only when the output sequence of the CPM system is converted into a binary sequence before transmission. This scenario is quite unnatural, as the typical reason for using a CPM with $M>2$ is that transmission is done directly using the symbol sequence. In this case, the aforementioned property for the probability distribution of CPM symbols does not hold. Nevertheless, this property is often implicity assumed to make the decoding process simple; see, e.g., $[7,9,10]$.

In this paper, we consider symbol-wise interleaving for SCCPM. More specifically, we provide appropriate criteria for interleaver designs and suitable decoding algorithms. Our approach is based on an analysis of the decoding errors associated with bitwise interleaving. We show that decoding errors can be better managed by using a symbol-wise interleaver, provided that the symbols for the outer code have a size of alphabet equal to $M$. Simulation results show that up to $1 \mathrm{~dB}$ improvements can be achieved in comparison with SCCPM schemes using bitwise interleaving. 
We note that the idea of using symbol-wise interleavers has been suggested in [12]-[16]. The outer code used in [12] is a non-binary code whereas in this paper, binary outer codes are used. This is an important distinction because non-binary codes are much more difficult to design in general. The outer codes used in [13]-[16] are binary. However, the decoding in $[13,14]$ is non-iterative. Also, the reason for using symbol-wise interleavers in $[15,16]$ is not clear and the advantages of the symbol-wise approach are not demonstrated. In addition, the symbol-wise interleaver proposed in this paper differs from those used in $[15,16]$ in that we introduce an additional bitwise swapping within each non-binary symbol. This does not affect symbol-wise operations in both coding and decoding, but it enhances the interleaving for the concatenation.

The paper is organized as follows. In section 2, we analyze the decoding errors associated with bitwise interleaving. In Section 3, we present a symbol-wise APP decoding structure. Section 4 introduce our SCCPM scheme. Section 5 shows some simulated results and compares our scheme and the SCCPM schemes using bitwise interleavers. Section 6 concludes the paper.

\section{DECODING ERROR ANALYSIS}

In this section, we consider SCCPM schemes with bitwise interleaving and analyze the decoding errors when $M>2$ and bitwise APP decoding is used.

The SCCPM scheme in consideration is shown in Fig. 1. It is formed by a binary outer code connected to a nonbinary CPM inner code through a bitwise interleaver $\Pi_{b}$ and a mapper used to form input symbols for the CPM. The communications channel is assumed to be subject to additive white Gaussian noise (AWGN).

Adopting notation similar to that in [18], an information sequence $U^{o}$ is fed into the outer encoder which outputs a coded symbol sequence $C^{o}$. Each of these symbols consists of $k_{0}$ bits. This sequence is expanded into a bit sequence and then interleaved into another bit sequence $\widetilde{C}^{o}$. The bit sequence $\widetilde{C}^{o}$ is then regrouped into a symbol sequence $U^{I}$ using a mapper which groups every $n_{0}$ bits into a symbol. That is, each CPM symbol has $n_{0}$ bits. The sequence $U^{I}$ is modulated to give the output symbol sequence $C^{I}$. For notational simplicity, we will drop the superscripts "I" and "O" whenever the constituent code is not confusing.

In the decoding process for either constituent code, let $\mathbf{P}_{i}(\cdot)$ and $\mathbf{P}_{o}(\cdot)$ represent the input and output probability distribution sequences of a constituent decoder, respectively, and $\mathbf{P}^{e}(\cdot)$ represents the extrinsic APP distribution sequence. The signal flows for the extrinsic APP distributions in the decoding process are illustrated in Fig. 2.

To understand the decoding errors in an iterative decoding process, we first consider the decoding error propaga- tion from the inner decoder to the outer decoder. The extrinsic APP distribution of a CPM symbol generated by the inner decoder at time $k$ is denoted by $P_{o}^{e}\left(u_{k}\right)$. Note that each CPM symbol $U_{k}$ is formed by $n_{0}$ bits, $U_{k}^{j}, j=1,2, \ldots, n_{0}$. Denote by $u_{k}^{j} \in\{0,1\}$ the realization of $U_{k}^{j}$. We have

$$
P_{o}^{e}\left(U_{k}=u_{k}\right)=P_{o}^{e}\left(U_{k}^{1}=u_{k}^{1}, U_{k}^{2}=u_{k}^{2}, \cdots, U_{k}^{n_{0}}=u_{k}^{n_{0}}\right)
$$

When transferring the symbol-wise APP into the APPs for the individual bits, we have

$$
P_{o}^{e}\left(U_{k}^{j}=u_{k}^{j}\right)=\sum_{U: U_{k}^{j}=u_{k}^{j}} P_{o}^{e}\left(U_{k}=u_{k}\right), j=1,2, \cdots, n_{0}
$$

After de-interleaving, these APPs will be split and then each of them will be regrouped with $k_{0}-1$ bitwise APPs from the symbols at times other than $k$, to form the a priori information of a symbol $C_{t}$ at some time $t$ in the outer decoding. For example, we suppose that an APP of bit $U_{k}^{j 1}$ from $U_{k}$ is regrouped with APPs of bits $U_{n}^{j 2}, U_{m}^{j 3}, \cdots, U_{q}^{j k_{0}}$ from symbols $U_{n}, U_{m}, \cdots, U_{q}$ with $n, m, \cdots, q \neq k$, respectively. Assuming all the bits forming a coded symbol $C_{t}$ in the outer code are statistically independent due to the interleaving, the a priori probability of the coded symbol in the outer code is given by

$$
\begin{aligned}
P_{i}\left(C_{t}=c_{t}\right)= & P_{i}\left(C_{t}^{1}=c_{t}^{1}, C_{t}^{2}=c_{t}^{2}, \cdots, C_{t}^{k_{0}}=c_{t}^{k_{0}}\right) \\
= & P_{i}^{e}\left(C_{t}^{1}=u_{k}^{j 1}\right) \cdot P_{i}^{e}\left(C_{t}^{2}=u_{n}^{j 2}\right) \cdots \\
& \left.\cdot P_{i}^{e}\left(C_{t}^{k_{0}}=u_{q}^{j k_{o}}\right)\right)
\end{aligned}
$$

It is apparent from the equation above that if one of the decoded symbols from the inner decoder is erroneous, then up to $n_{0}$ symbols for the outer code are also erroneous, causing possible serious error propagations.

Now we consider the possible decoding errors caused by the transfer of the APPs from the outer decoder to the inner decoder. If the output sequence of the inner code were expanded into a bit sequence before transmission, then all the bits in the sequence could be regarded as independent. In this case, the a priori information about a CPM symbol could be calculated from a group of the APPs of $n_{0}$ bits (which are passed from the outer decoder through the interleaver) by

$$
P_{i}\left(U_{k}=u_{k}\right)=\prod_{j} P_{i}\left(U_{k}^{j}=u_{k}^{j}\right)
$$

where $j=1,2, \cdots, n_{0}$. However, the reality is that the non-binary CPM symbols are directly transmitted. It has been pointed out in $[18,9]$ that the equation above is not valid in this case. This is due to the fact that the $n_{0}$ bits in a CPM symbol are subject to the same noise samples and the independence assumption of these $n_{0}$ bits does not hold anymore. However, this product is often implicitly used in 


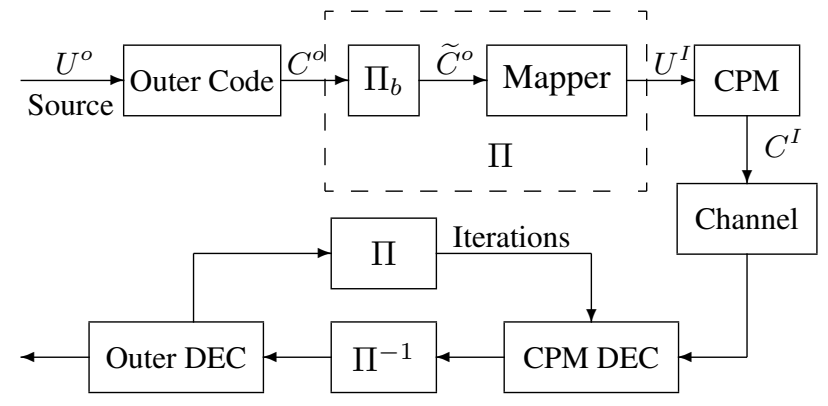

Fig. 1. Bitwise Interleaving Serial Concatenated CPM Scheme

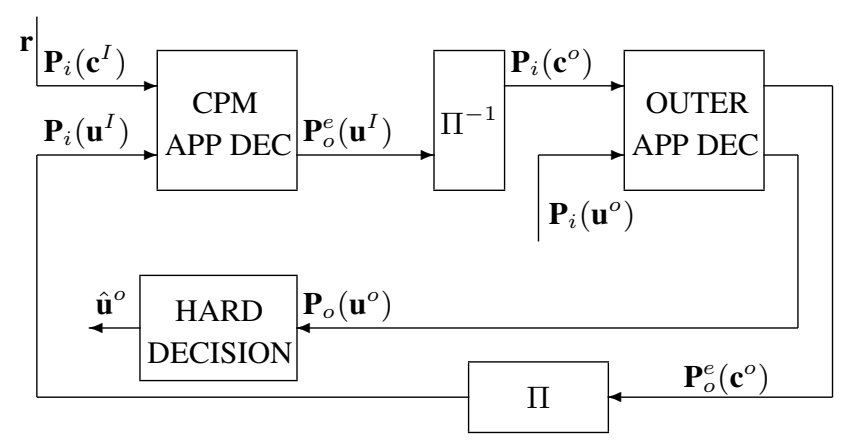

Fig. 2. Iterative Soft Decision Decoding

bitwise decoding, see, e.g., [7, 9]. Since our simulation results are in favor of symbol-wise interleaving, the use of this assumption, which is invalid for non-binary CPM, is likely a cause for the performance degradation for the bitwise approach.

\section{SYMBOL-WISE APP DECODING}

In this section, we study the possibility of symbol-wise APP decoding.

¿From the analysis in the previous section, we see the decoding performance is degraded by two factors: 1) the splitting of a CPM symbol and regrouping of $k_{0}$ bits from different CPM symbols; 2) the invalid assumption of statistical independence in (2). Bitwise operations are necessary when $k_{0} \neq n_{0}$, and in this case, bitwise decoding is needed.

However, symbol-wise APP decoding is possible when $k_{0}=n_{0}$. Indeed, based on the results in [18], the symbolwise APPs $\widetilde{P}_{o}\left(c_{k}\right)$ and $\widetilde{P}_{o}\left(u_{k}\right)$ can be calculated by

$$
\begin{gathered}
\widetilde{P}_{o}\left(c_{k}\right)=\sum_{e: C_{k}(e)=c_{k}} \alpha_{k-1}\left[s^{S}(e)\right] P_{i}\left(u_{k}(e)\right) P_{i}\left(c_{k}(e)\right) \beta_{k}\left[s^{E}(e)\right] \\
\widetilde{P}_{o}\left(u_{k}\right)=\sum_{e: U_{k}(e)=u_{k}} \alpha_{k-1}\left[s^{S}(e)\right] P_{i}\left(u_{k}(e)\right) P_{i}\left(c_{k}(e)\right) \beta_{k}\left[s^{E}(e)\right]
\end{gathered}
$$

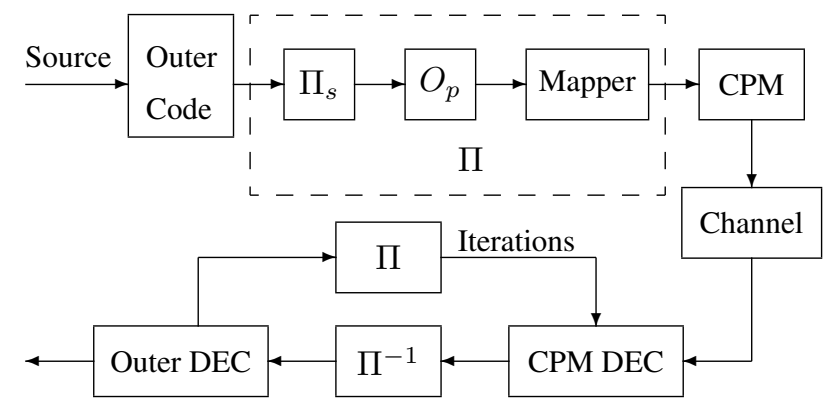

Fig. 3. Symbol-wise Interleaving Serial Concatenated CPM Scheme

respectively, where

$$
\begin{aligned}
& \alpha_{k}(s)=\sum_{e: s^{S}(e)=s} \alpha_{k-1}\left[s^{S}(e)\right] P_{i}\left(u_{k}(e)\right) P_{i}\left(c_{k}(e)\right) \\
& \beta_{k}(s)=\sum_{e: s^{S}(e)=s} \beta_{k+1}\left[s^{S}(e)\right] P_{i}\left(u_{k+1}(e)\right) P_{i}\left(c_{k+1}(e)\right)
\end{aligned}
$$

and we have ignored the normalization constants for simplicity. Note, also, the corresponding extrinsic APPs are $P_{o}^{e}\left(c_{k}\right)=\widetilde{P}_{o}\left(c_{k}\right) / P_{i}\left(c_{k}\right)$ and $P_{o}^{e}\left(u_{k}\right)=\widetilde{P}_{o}\left(u_{k}\right) / P_{i}\left(u_{k}\right)$, respectively. Expressions similar to $(3) \sim(6)$ can also be found in $[9,16]$.

Observe that there are no bitwise operations in (3) (6). The only possible bitwise operations are within the interleaving and de-interleaving. When $k_{0}=n_{0}$, we can use a symbol-wise interleaver which maps each $n_{0}$ bits of input to $n_{0}$ bits of output. The only disadvantage of using symbol-wise interleavers is that the effective interleaver size becomes $1 / n_{0}$-th of a bitwise interleaver. But we will see that this disadvantaged is overweighed by its advantages. Note also that the need for $k_{0}=n_{0}=\log _{2} M$ is somewhat restrictive in that the outer code rate dictates the value of $M$.

\section{THE PROPOSED SCCPM}

The proposed symbol-wise interleaving scheme shown in Fig. 3 is identical to the bitwise interleaving scheme in Fig. 1 except for two changes. Firstly, the interleaver $\Pi_{s}$ operates in a symbol-wise fashion. Secondly, after symbolwise interleaving, the $n_{0}$ bits within each symbol are then interleaved by $O_{p}$. The output of this operation is then further converted using a Natural Binary Code (NBC) or Gray mapper before being sent to the CPM. We choose the symbol-wise interleaver to be an $S$-random interleaver (in a symbol-wise sense).

In this paper, we investigate two types of SCCPM schemes. The first type is a coded quaternary CPM which uses as the 
outer code a rate- $1 / 2$ nonrecursive convolutional code with the generator matrix $G(D)=\left[1+D+D^{2}, 1+D^{2}\right]$ or $(7,5)$ in octal representation. The second type is a coded octal CPM which uses a rate- $2 / 3$ convolutional code as an outer code. The generator matrix for the outer code is given by

$$
G(D)=\left[\begin{array}{ccc}
1+D & D & 1+D \\
D & 1 & 1
\end{array}\right]
$$

We choose these schemes because they have been used by several researchers; see, e.g., [7].

Although the use of symbol-wise interleaving implies that the individual bits in different symbols are not mixed and regrouped, the ordering of the $n_{0}$ bits within a symbol has some influence on the decoding performance. Thus, when discussing a code with symbol-wise interleaving, we also need to make it explicit the ordering pattern $O_{p}$ of the $n_{0}$ bits within a coded symbol. Obviously, $O_{p}$ is nothing but an interleaver of the size $n_{0}$. Given other parameters in a SCCPM, $O_{p}$ can be optimized to give the best performance. The choices for $O_{p}$ can be fixed, random or periodic.

\section{SIMULATIONS AND COMPARISONS}

In this section, we present and compare some simulation results when AWGN channels and coherent demodulations are assumed. For each comparison, both the bitwise and symbol-wise schemes use the same CPM, the same overall code rate and same input block size. The comparison is performed on the power efficiency at $\mathrm{BER}=10^{-5}$. For brevity, $\mathrm{CPM}$ using the raised cosine shape function with duration $L$ is represented by $L R C$ CPM.

The simulated results for the serially concatenated quaternary $2 R C$ and $3 R C \mathrm{CPMs}$ are illustrated in Fig. 4. These schemes use $p=4$, input block size $N=4096$, and the outer codes are rate-1/2 $(7,5)$ codes. We see from the figure that, for bitwise interleaving, Gray Mapping gives a better result than NBC Mapping. This observation is actually different from what is conjectured in [7]. When $O_{p}=[21]$, along with the NBC mapping, the symbol-wise schemes for $2 R C$ and $3 R C$ give $0.4 \mathrm{~dB}$ and $0.8 \mathrm{~dB}$ improvements, respectively, when compared with their bitwise counterparts (with Gray Mapping). The results of the coded octal 3RC schemes are shown in Fig. 5. These are the cases corresponding to $p=4, N=1024$, and the rate- $2 / 3$ outer code generator matrix as stated in Section 4. The best result is given by the scheme using symbol-wise interleaving, the circled solid curve. For this scheme, $O_{p}$ is chosen randomly from the set $\left\{\left[\begin{array}{lll}1 & 2 & 3\end{array}\right],\left[\begin{array}{lll}2 & 1 & 3\end{array}\right],\left[\begin{array}{lll}2 & 3 & 1\end{array}\right]\right\}$, and NBC mapping is used. This result is $0.75 \mathrm{~dB}$ better than that of the best bitwise scheme which uses Gray Mapping. Generally speaking, for the same values of $L$ and $M$, a larger value of $p$ usually leads to a larger gain when symbol-wise interleaving is used. When $p$ is large, a low order outer code is usually

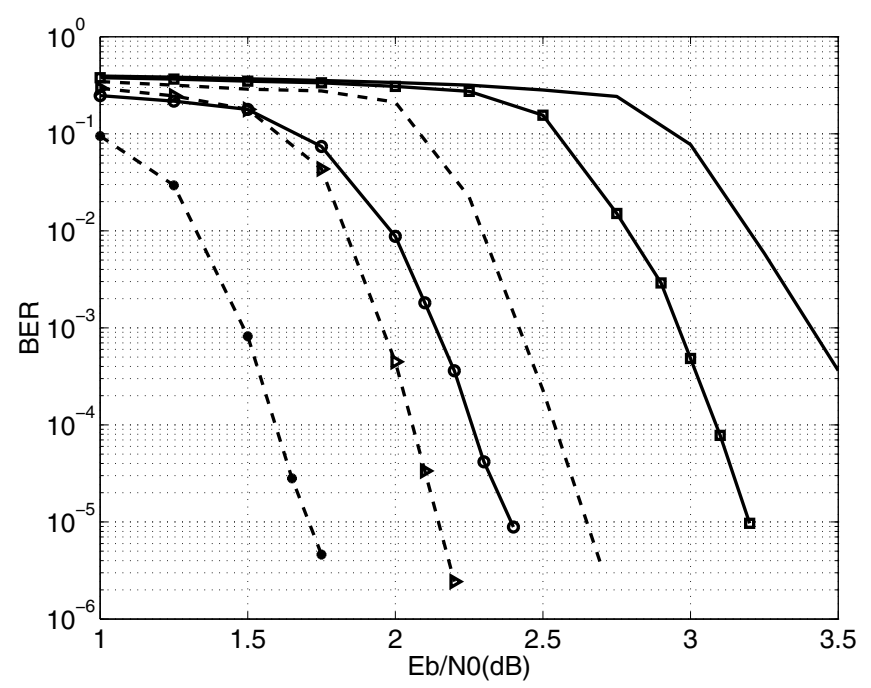

Fig. 4. Simulation results of the coded quaternary CPMs with rate- $1 / 2$ outer code, $p=$ $4, N=4096$ and 15 decoding iterations. Legends: solid - bitwise, coded 3RC CPM, NBC; squared solid - bitwise, coded 3RC CPM, Gray; circled solid - symbol-wise, coded 3RC CPM, NBC; dash - bitwise, coded 2RC CPM, NBC; triangulated dash -- bitwise, coded 2RC CPM, Gray; stared dash —- symbol-wise, coded 2RC CPM, NBC

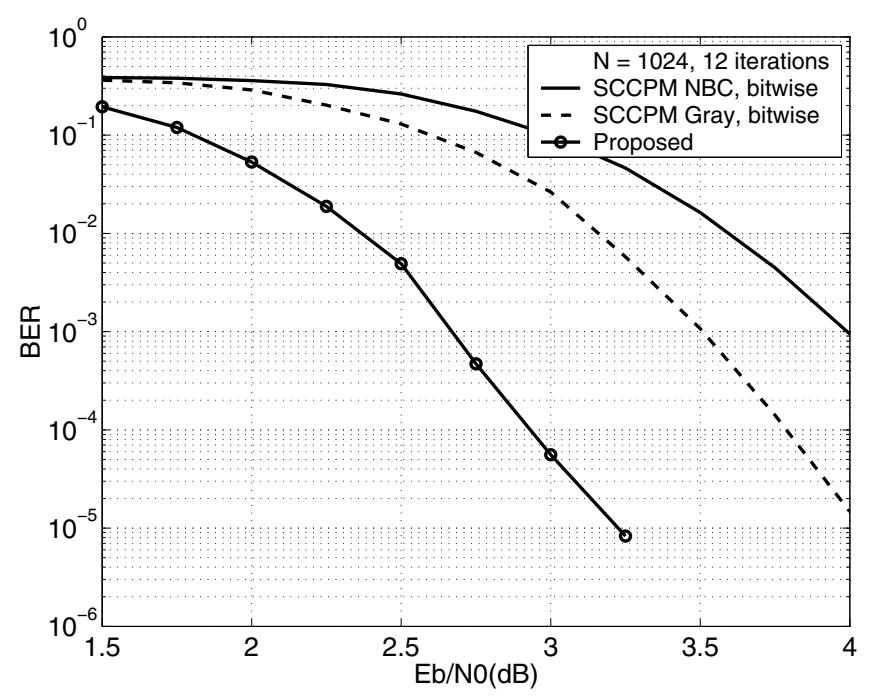

Fig. 5. Simulation results of the coded octal 3RC CPMs with rate- $2 / 3$ outer code, $p=4, N=1024$ and 12 decoding iterations 
preferred. Typically, the code $(7,5)$ gives the best performance. However, when $p$ is small, more improvement may be achieved by using a higher order outer code. A typical example is shown in Fig. 6. The simulated schemes are the coded quaternary 3RC CPMs with $p=2$ and $N=1024$. The best bitwise interleaving scheme is given by using the $(7,5)$ outer code with Gray Mapping. For symbol-wise interleaving, using the $(7,5)$ outer code with $O_{p}=[21]$ with a NBC Mapping gives a gain of about $0.35 \mathrm{~dB}$, whereas using the $(15,17)$ outer code with NBC Mapping and $O_{p}$ randomly chosen from the set $\left\{\left[\begin{array}{ll}1 & 2\end{array}\right],\left[\begin{array}{ll}2 & 1\end{array}\right]\right\}$ offers about $0.6 \mathrm{~dB}$ improvement. Another interesting example is shown in Fig. 7. The simulated schemes are the coded quaternary 2RC CPMs with $p=2$ and $N=1024$. When the outer code is $(7,5)$, the result for symbol-wise interleaving is actually worse than that of its bitwise counterpart. However, when the outer code generator is $(53,75)$, a gain of $0.5 \mathrm{~dB}$ is obtained by the symbol-wise scheme compared with the bitwise scheme with the outer code $(7,5)$. Note that for both examples above, high order outer codes do not offer any improvement for bitwise interleaving.

We have also tried the serially concatenated quaternary 3RC CPM for $p=2,3,4,5, N=1024$ and rate- $1 / 2(7,5)$ outer code. For $p=2,4,5, O_{p}=\left[\begin{array}{ll}2 & 1\end{array}\right]$ and NBC mapping give the best result. For $p=3, O_{p}=\left[\begin{array}{ll}1 & 2\end{array}\right]$ and Gray mapping turns out to be better. Simulation results show that improvements of 0.5 to $1 \mathrm{~dB}$ are obtained by using the proposed schemes against their best bitwise counterparts (all with Gray Mapping). The simulation results are not shown due to page limits. Additional simulations show that small improvements can be achieved for other SCCPMs including 4CPFSK and 8CPFSK with $N=1024, h=1 / 4$ and $1 / 8$. Therefore, we conclude that the proposed symbolwise interleaving approach outperforms the bitwise interleaving for a large class of SCCPM systems.

\section{CONCLUSION}

In this paper, we have demonstrated the advantage of using symbol-wise interleavers for SCCPM designs. A suitable APP decoding scheme for this type of SCCPM has also be presented. The symbol-wise approach is applicable to any SCCPM with a binary outer code, provided that the outer coded symbols and the CPM symbols have the same alphabet size. Simulation results show that for many cases the proposed symbol-wise scheme outperforms their bitwise counterparts in terms of power-bandwidth efficiency, with the same or similar computational complexities.

\section{REFERENCES}

[1] J. B. Anderson, T. Aulin and C. E. Sundberg, Digital Phase Modulation, Plenum, New York, 1986.

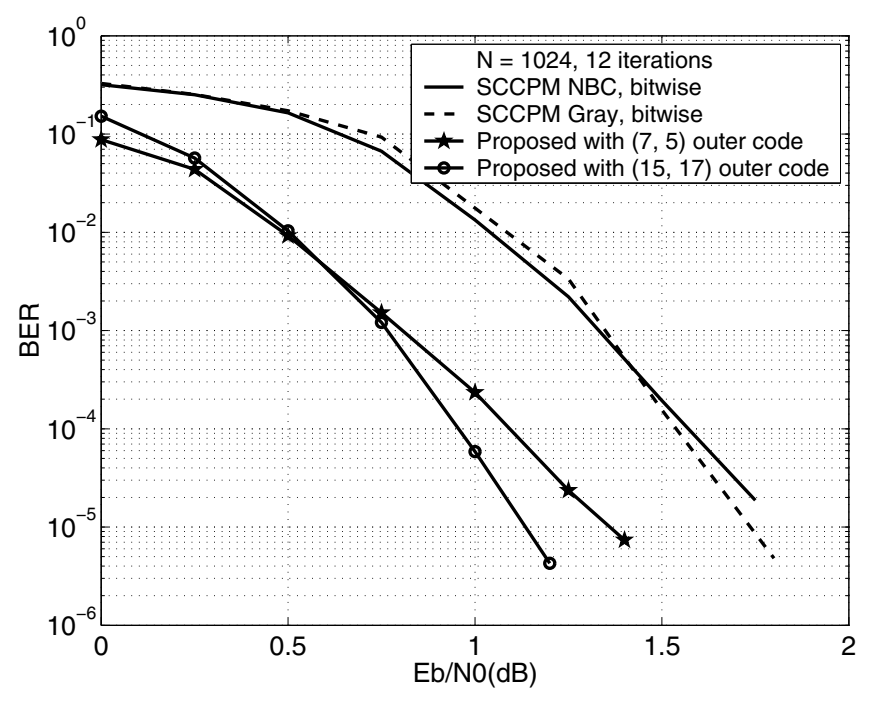

Fig. 6. Simulation results of the coded quaternary $3 \mathrm{RC}$ CPMs with rate- $1 / 2$ outer code, $p=2, N=1024$ and 12 decoding iterations

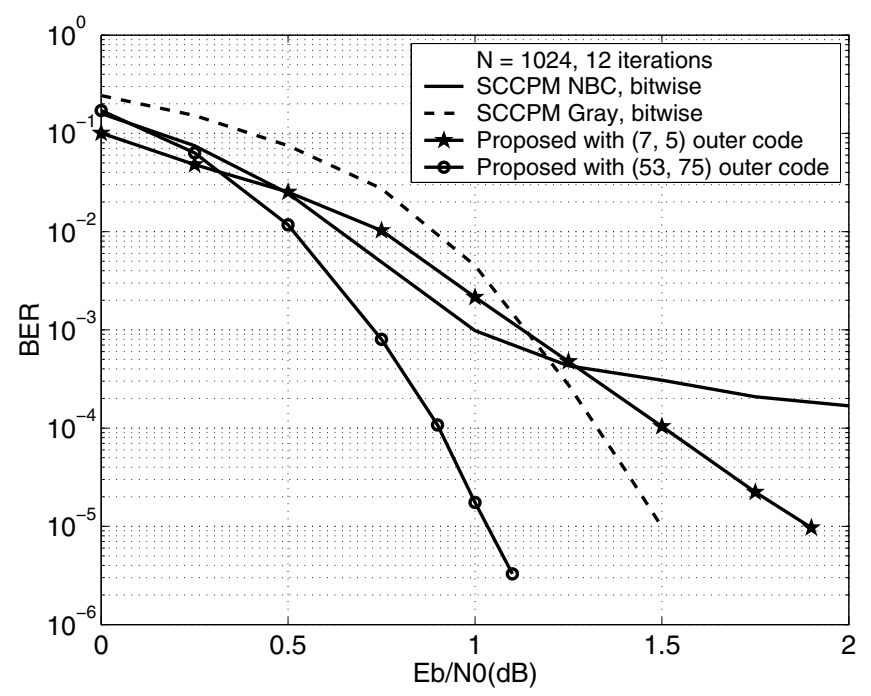

Fig. 7. Simulation results of the coded quaternary $2 \mathrm{RC}$ CPMs with rate- $1 / 2$ outer code, $p=2, N=1024$ and 12 decoding iterations 
[2] J. G. Proakis, Digital Communications, 4th ed., McGraw Hill, 2001.

[3] B. E. Rimoldi and Q. Li, "Coded continuous phase modulation using ring convolutional codes," IEEE Trans. Commun., vol. 43, no. 11, pp. 2714-720, 1995.

[4] B. Rimoldi, "Design of coded CPFSK modulation systems for bandwidth and energy efficiency ," IEEE Trans. Commun., vol. 37, no. 9, pp. 897-905, 1989.

[5] — "A decomposition approach to CPM," IEEE Trans. Inform. Theory, vol. 34, pp. 260-270, 1988.

[6] S. V. Pizzi and S. G. Wilson, "Convolutional coding combined with Continuous Phase Modulation," IEEE Trans. Commun., vol. 33, no. 1, pp. 20-29, 1985.

[7] P. Moqvist and T. Aulin, "Power and Bandwidth Efficient Serially concatnated CPM with iterative decoding, " GLOBECOM'OO. IEEE, volume: 2, 27 Nov. -1 Dec. 2000, pages: $790-794$ vol. 2.

[8] _ - "Serially concatnated continuous phase modulation with iterative decoding, " IEEE Trans. Commun., vol. 49, no. 11, pp. 1901-1915, 2001.

[9] P. Moqvist, "Serially concatnated Systems: An Iterative Decoding Approach with Application to Continuous Phase Modulation, " Lic. Eng. Thesis, Dept. of Comp.Eng., Chalmers Univ. of Techn., Göteborg.

[10] K. R. Narayanan and G. L. Stüber, "Performance of trellis-coded CPM with iterative demodulation and decoding," IEEE Trans. Commun., vol. 49, pp. 676687, 2001.

[11] M. Singh and I. J. Wassell, "Comparison between serial and parallel concatenated channel coding schemes using continuous phase modulation over AWGN and fading channels," Int. Conf. Comms., Las Vegas, June 2001.

[12] - "Effective channel coding of serially concatennated encoders and CPM over AWGN and Rician channels," MILCOM 2000 , vol. 1, pp. 402-406, 2000.

[13] R.W. Kerr and P.J. McLane, "Cohernt Detection of Interleaved Trellis Encoded CPFSK on Shadowed Mobile Satellite Channels," IEEE Trans. Veh. Technol, vol. 41, pp. 159-169, 1992.

[14] L. Yiin and G.L. Stüber, "Error Probability of Cohernt Detection for Trellis Coded Partial Response CPM on Rician Fading Channels," IEEE Trans. Veh. Technol, vol. 45, pp. 358-363, 1996.
[15] X. Zhang and M. P. Fitz, "Soft-output demodulator in space-time coded continuous phase modulation," IEEE trans. on Signal Proc., Vol. 50, pp. 2589-2598, 2002.

[16] — - "Soft Output Diversity Combining for CPM Signals over Space-time Correlated Rayleigh Fading Channels," ICC'01 , pp. 580-585, 2001.

[17] S. Benedetto, D. Divsalar, G. Montorsi and F. Pollara, "Serial concatenation of interleaved codes: performance analysis, design, and iterative decoding," IEEE Trans. Inform. Theory, vol. 44, pp. 909-926, 1998.

[18] —, "A soft-input soft-output APP module for iterative decoding of concatenated codes," IEEE Commun. Lett., vol. 1, pp. 22-24, 1997. 